\title{
El hábito de fumar se asocia a baja concentración plasmática de hormona antimülleriana en mujeres infértiles
}

\author{
ARIEL FUENTES, JAVIER ESCALONA, PABLO CÉSPEDES, \\ VICTORIA REPETTO, GERMAN IÑIGUEZ
}

\section{Effects of smoking on plasma antimüllerian hormone concentrations among infertile women}

\begin{abstract}
Background: Smoking may hamper female fertility, probably modifying ovarian reserve. Antimüllerian hormone (AMH) is an accurate marker for ovarian reserve. Aim: To look for an association between smoking status and plasma AMH concentration. Patients and Methods: A cohort of 141 infertile women in a university setting in Santiago, Chile was studied. Demographic and smoking data, including the number of cigarettes smoked during the last week, were collected. A blood sample was obtained and kept frozen until determination of AMH by ELISA and follicle stimulating hormone (FSH) and estradiol at day three of the menstrual cycle, by radioimmunoanalysis. Results: Thirty two participants smoked (23\%). There were no significant differences in age, parity, body mass index, causes of infertility and day three FSH and estradiol between smokers and nonsmokers. According to a regression analysis, there was a significant decrease in $A M H$ concentration with age and active cigarette smoking. A drop in AMH of $-0.189 \mathrm{ng} / \mathrm{mL}$ with a unitary change in age and a decrease of $-2.29 \mathrm{ng} / \mathrm{mL}$ when everything else remains constant, except the smoking status, were established $(p<0.001$ and $r 2=0.134)$. However, no dose response was observed when the number of cigarettes smoked during the last week were introduced in the model. Furthermore, no significant association of plasma AMH with day three plasma FSH and estradiol concentrations was observed. Conclusions: Cigarette smoking is associated with decreased AMH plasma concentrations among infertile women. However there was no dose response relationship. The mechanisms underlying this association are unknown and further investigation is required.
\end{abstract}

(Rev Med Chile 2013; 141: 23-27).

Key words: Age factors; Anti-Mullerian hormone; Infertility; Smoking.

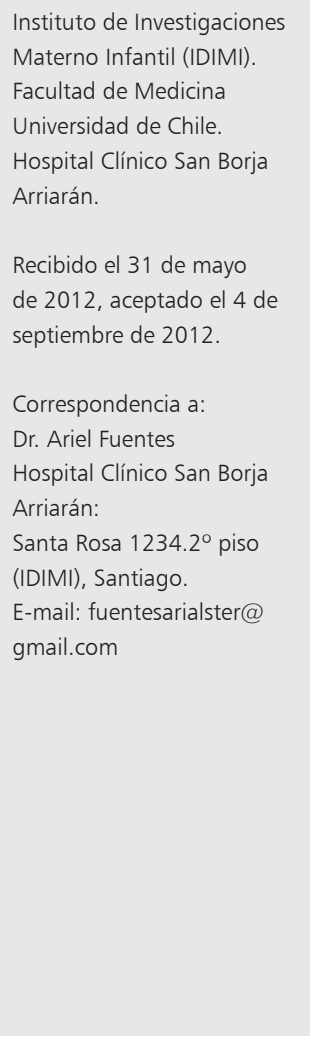

Instituto de Investigaciones Materno Infantil (IDIMI). Facultad de Medicina Universidad de Chile. Hospital Clínico San Borja Arriarán.

Recibido el 31 de mayo de 2012, aceptado el 4 de septiembre de 2012.

Correspondencia a: Dr. Ariel Fuentes Hospital Clínico San Borja Arriarán:

Santa Rosa $1234.2^{\circ}$ piso (IDIMI), Santiago E-mail:fuentesarialster@ gmail.com

\begin{abstract}
S e ha descrito que el hábito de fumar tiene un impacto negativo sobre los resultados reproductivos tales como la frecuencia de aborto, embarazo ectópico, la fecundabilidad y la edad de ocurrencia de la menopausia. En promedio las mujeres fumadoras tienen una menopausia adelantada en un año respecto de sus pares no fumadoras $^{1,2}$. Sin embargo, los mecanismos a través de los cuales el cigarrillo adelanta el final de la vida reproductiva se desconocen.
\end{abstract}

Ha sido comunicado que en la mujer el ciga- rrillo aumenta los niveles de hormona folículo estimulante (FSH) que ha sido propuesta como marcadora de la reserva ovárica ${ }^{3}$. Debido a que FSH es producida en la hipófisis y no en el ovario, este efecto pudiera derivar de una acción directa sobre el gonadotropo hipofisiario sin reflejar necesariamente la cuantía del pool remanente de ovocitos en el ovario.

La hormona antimülleriana (AMH) se expresa primariamente en las células de granulosa del folículo secundario en crecimiento, preantral y antral 
temprano. Así, AMH es un marcador directo de la reserva ovárica ${ }^{4,5}$. Estudios en animales indican que los niveles de esta hormona se correlacionan en forma directa con el número de folículos en crecimiento y el número de folículos primordiales $^{6}$. Por otra parte, se sabe que la AMH no varía significativamente a lo largo del ciclo menstrual o con los cambios cíclicos de las gonadotrofinas hipofisiarias ${ }^{7,8}$. El objetivo del presente trabajo fue determinar la existencia de una asociación negativa entre el hábito de fumar y los niveles circulantes de hormona antimülleriana. Por lo tanto, la hipótesis de este estudio sostiene que el consumo de cigarrillos se asocia a niveles disminuidos de AMH.

\section{Material y Método}

La población incluida en este estudio comprendió 146 mujeres que consultaron por infertilidad en el Instituto Investigaciones Materno Infantil de la Facultad de Medicina de la Universidad de Chile (IDIMI) en Santiago, durante el año 2010. Se excluyeron las mujeres con antecedente de síndrome de ovario poliquístico por su conocido aumento de los niveles circulantes de $\mathrm{AMH}$ y las que habían sido sometidas a cirugía ovárica por endometriosis u otras causas. Por otra parte, se incluyeron sólo parejas en que el factor masculino presentaba parámetros seminales normales.

A todas las participantes se les realizó medición de la AMH sérica, FSH y estradiol en el día 3 de su ciclo menstrual, considerando el día 1 como le primer día de su menstruación.

La condición de fumadora se estableció en base a autoreporte a través de la contestación de un cuestionario utilizado y validado previamente en un estudio de este grupo ${ }^{9}$. Brevemente, este instrumento estableció y cuantificó la relación actual del individuo con el hábito de fumar (al momento de contestar el cuestionario) y su relación con el cigarrillo a lo largo de la vida. En este estudio se utilizó como indicador el número de cigarrillos consumidos durante la semana previa a la aplicación del cuestionario. Se consideró fumadora para los fines de este estudio a las mujeres que declararon haber fumado la semana anterior.

\section{Determinación de AMH}

La determinación de la concentración de AMH se estableció mediante inmunoensayo ELISA
(Disgnostic System, laboratorio, Inc Beckman coulter, Webster, TX. especifico Estados Unidos de Norteamérica). Los resultados se expresaron en ng/ $\mathrm{ml}$. El límite inferior detectable para AMH fue de $0,05 \mathrm{ng} / \mathrm{ml}$ y sus coeficientes intra e inter-ensayo fueron 3,4 y $6,5 \%$ respectivamente.

\section{Determinación de FSH}

La determinación de la concentración de FSH se estableció mediante ensayo inmunométrico, los resultados fueron expresados en $\mathrm{mUI} / \mathrm{ml}$ y el límite inferior detectable para esta hormona fue de $0,06 \mathrm{mIU} / \mathrm{ml}$. Los coeficientes intra e inter-ensayo fueron de 2,9 y $4,9 \%$, respectivamente.

\section{Determinación de estradiol}

Las concentraciones de estradiol se establecieron mediante radioinmunoensayo, los resultados fueron expresados en $\mathrm{pg} / \mathrm{ml}$. El mínimo nivel detectable para este esteroide fue de $8 \mathrm{pg} / \mathrm{ml} \mathrm{y}$ los coeficientes intra e inter-ensayo 5,3 y $6,4 \%$ respectivamente.

\section{Cuestionario}

A todas las participantes se les aplicó un cuestionario en el que se preguntó por datos demográficos como la edad y la paridad. Además se averiguó sobre las causas de infertilidad y el consumo actual y pasado de cigarrillos con énfasis en la dosis de cigarrillos fumados durante la última semana.

\section{Análisis estadístico}

El cálculo del n muestral se basó en una diferencia entre las medias de $0,7 \mathrm{ng} / \mathrm{ml}$ de $\mathrm{AMH}$ con una desviación estándar de $0,91 \mathrm{ng} / \mathrm{ml}$ de acuerdo a datos piloto de nuestro laboratorio. Así, el número mínimo de sujetos por grupo necesarios para este estudio fue calculado en 30 sujetos, asumiendo una tasa de 3,5 controles por cada caso.

La distribución de variables continuas se estableció usando el test de Kolmogorov - Smirnov. Para la comparación de medias se usó el test de $\mathrm{t}$ de Student. Se empleó regresión lineal para establecer el efecto de la edad sobre la concentración AMH ajustada por la condición de fumador. Los resultados son expresados como la media \pm el error típico de la media.

\section{Resultados}

Ingresaron al estudio 146 pacientes observándose una prevalencia del hábito de fumar cigarri- 
Tabla 1. Características de la población estudiada

\begin{tabular}{|lcc|}
\hline & $\begin{array}{c}\text { Fumadoras } \\
(\mathbf{n}=\mathbf{3 2})\end{array}$ & $\begin{array}{c}\text { No fumadoras } \\
(\mathbf{n}=\mathbf{1 1 4})\end{array}$ \\
\hline Edad & $38 \pm 0,85$ & $36,64 \pm 0,56$ \\
$\mathrm{IMC}$ & $26 \pm 0,32$ & $26,8 \pm 0,62$ \\
$\mathrm{FSH}^{* *}$ & $14,86 \pm 2,33$ & $14,03 \pm 2,95$ \\
\hline Estradiol** & $28,89 \pm 5,12$ & $30,67 \pm 3,62$ \\
\hline
\end{tabular}

*Valores corresponden a la media \pm Error típico de la media.

**FSH y Estradiol tomados en día 3 del ciclo.
Tabla 2. Causas de infertilidad

\begin{tabular}{|lccccc|}
\hline Causa & $\begin{array}{c}\text { No fumadoras } \\
\text { (n) }\end{array}$ & $\begin{array}{c}\text { Fumadoras } \\
\text { (n) }\end{array}$ & $\begin{array}{c}\text { Total } \\
\text { (n) }\end{array}$ \\
\hline Factor tubario & 45 & 78 & 13 & 22 & 58 \\
Endometriosis & 35 & 79 & 9 & 21 & 44 \\
Anovulación & 20 & 80 & 5 & 16 & 25 \\
Otras & 14 & 74 & 5 & 26 & 19 \\
\hline Total & 114 & & 32 & & 146 \\
\hline
\end{tabular}

Tabla 3. Regresión Lineal: Edad y status de fumador sobre concentraciones de AMH sérica

\begin{tabular}{|lccccc|}
\hline Modelo & $\begin{array}{c}\text { Coeficientes no estandarizados } \\
\text { Beta }\end{array}$ & $\begin{array}{c}\text { Coeficientes tipificados } \\
\text { Error standard }\end{array}$ & t & Peta \\
\hline Intercepto & 10,587 & 2,182 & & 4,853 &, 000 \\
1 Edad & $-0,134$ & 0,057 & $-0,189$ & $-2,360$ &, 020 \\
\hline Condición de fumador & $-2,288$ & 0,642 & $-0,285$ & $-3,561$ &, 001 \\
\hline
\end{tabular}

a. Variable dependiente: AMH. $r=0,322 ; r^{2}=0,103$

llos de 23\% $(\mathrm{n}=32)$. Como se observa en la Tabla 1 no hubo diferencias entre fumadoras y no fumadoras respecto a la media de edad, índice de masa corporal (IMC), concentración de FSH ni estradiol en día 3 del ciclo menstrual. La causa más frecuente de infertilidad correspondió a alteraciones de las trompas de Falopio seguidas por endometriosis, anovulación e infertilidad de causa desconocida (Tabla 2). No se observó diferencias significativas en la frecuencia de estas causas de infertilidad femenina entre la población de mujeres fumadoras y no fumadoras.

Las mujeres fumadoras consumieron 30,69 $\pm 5,22$ cigarrillos a la semana (mínimo 7 máximo de 140) en promedio.

En un análisis bivariado, la concentración media de AMH de la población total fue de $2,8 \mathrm{ng} / \mathrm{ml}$, correspondiendo una media de $3,23 \pm 0,33 \mathrm{ng} / \mathrm{ml}$ a las no fumadoras y de $0,92 \pm 0,14 \mathrm{ng} / \mathrm{ml}$ a las pacientes fumadoras (Figura 1).

Tal como se observa en la Tabla 3 en el análisis multivariado la regresión lineal demostró que tanto la edad como el hábito de fumar cigarrillos tienen un

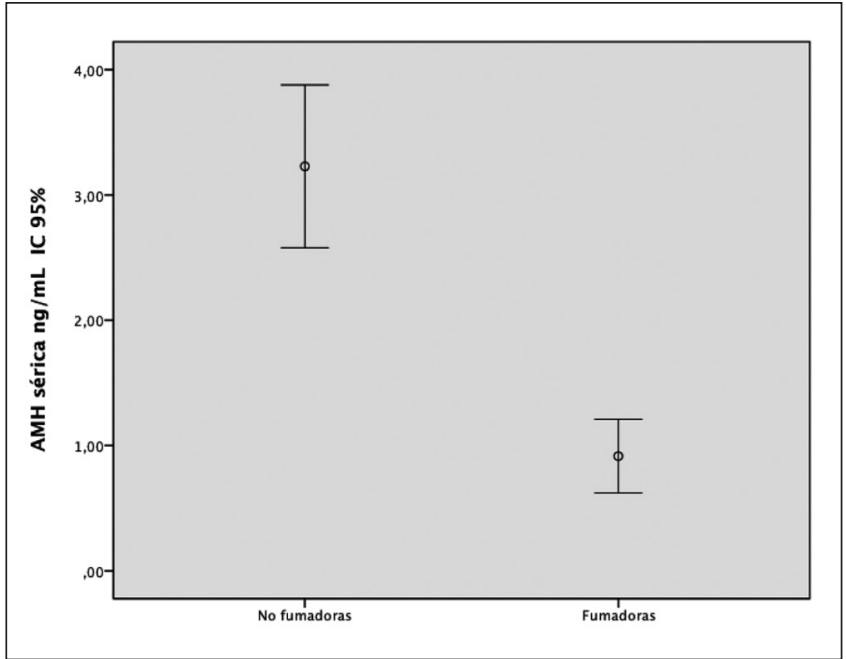

Figura 1. Niveles de AMH en fumadoras y no fumadoras. Los círculos señalan el valor de la AMH. Los círculos señalan el valor de la media y las barras los intervalos de confianza de 95\%.

efecto significativo sobre la concentración plasmática de $\mathrm{AMH}$, asociándose ambas variables con una caída de las concentraciones de $\mathrm{AMH}$ en forma significativa, con un $\mathrm{R}=-0,322$ y un $\mathrm{r}^{2}=0,103(\mathrm{p}<0,05)$.

Una correcta interpretación de la Tabla 1 indica que mientras todo lo demás permanece constante por cada año 
que la mujer aumenta en edad, AMH desciende en $-0,189 \mathrm{ng} / \mathrm{mL}$. Además, a edades iguales al ir de una mujer no fumadora a una fumadora se produce un cambio de $-2,288 \mathrm{ng} / \mathrm{mL}$ en la concentración de AMH plasmática.

Al incorporar al modelo el número de cigarrillos consumidos en la semana anterior a la aplicación del cuestionario no se observó una asociación significativa con cambios en los niveles de $\mathrm{AMH}$, lo que descarta una relación dosis respuesta. Tampoco se observó una asociación significativa con los niveles de FSH, estradiol en día 3 del ciclo menstrual ni la causa de la infertilidad.

\section{Discusión}

La prevalencia del hábito de fumar al momento de realizarse este estudio en la población femenina infértil fue de 23\% muy similar al 19\% comunicado recientemente por nuestro grupo ${ }^{9}$. Nuestros resultados avalan la asociación del hábito de fumar con una caída de las concentraciones de AMH reportada por otros autores ${ }^{10,11,12}$. Igualmente importante, estos resultados demuestran que $\mathrm{AMH}$ es un marcador de reserva ovárica que tiende a disminuir a medida que la mujer envejece y es sensible a factores ambientales. Esta disminución de la AMH circulante a lo largo del tiempo no es influida por las variaciones que experimentan las gonadotropinas durante el ciclo menstrual, especialmente la FSH, hormona que sufre una fuerte alza al final de la vida reproductiva. De ello se puede deducir que AMH representa un marcador de reserva ovárica en una etapa bastante más precoz comparada con $\mathrm{FSH}^{13}$.

Sin embargo, este estudio, como otros ${ }^{14}$, falló para establecer una relación dosis respuesta al correlacionar el número de cigarrillos fumados en la semana anterior a la aplicación del cuestionario con los niveles de AMH plasmática encontrados. Resultados similares han sido reportados por Freour ${ }^{15}$.

Por otra parte, no están claros los mecanismos a través de los cuales el cigarrillo puede inducir esta caída en los niveles plasmáticos de AMH. Las células de granulosa de los folículos preantrales y antrales tempranos son responsables de la síntesis y liberación al torrente sanguíneo de la AMH. El humo de cigarrillo puede ejercer una acción inhibitoria de la proliferación o estimula- dora de la apoptosis de estas células de granulosa. Así, al disminuir la masa crítica de este tipo de células sería esperable una caída en la síntesis y liberación de la AMH. El estudio de la influencia del humo de cigarrillo sobre la proliferación y apoptosis celular en la población de células de granulosa es un punto clave en la dilucidación de esta hipótesis. Los actuales resultados respecto de la concentración de AMH en plasma son muy similares a los encontrados por nuestro grupo en líquido folicular'. En ese estudio se observó una concentración de AMH significativamente menor entre las fumadoras medidas en el fluido folicular y se descartó la presencia de interacción estadística entre edad y consumo de cigarrillos. Así, ambos estudios demuestran que tanto la edad como el hábito de fumar se asocian significativamente con una caída de las concentraciones de hormona antimülleriana.

Una de las fortalezas del presente estudio consiste en el manejo de las principales variables generadoras de confusión como la edad y el índice de masa corporal. Así, la principal conclusión de este estudio es que tanto la edad como la condición de fumadora juegan un rol, estadísticamente significativo aunque modesto cuantitativamente, sobre las concentraciones plasmáticas de $\mathrm{AMH}$.

Este estudio tiene algunas limitaciones como por ejemplo el escaso número de fumadoras cuya condición de tales se basa exclusivamente en el cuestionario autoadministrado. Es posible que algunas mujeres no se muestren proclives a reconocer que fuman conduciendo a una subestimación de la prevalencia del consumo de tabaco. Por otra parte, no se intentó en este trabajo obtener datos acerca de la condición de fumadora pasiva. Así, si algunas de ellas fueron mal calificadas como no fumadoras esto puede haber debilitado la asociación entre cigarrillo y concentraciones de AMH.

A pesar de que los estudios de corte transversal que han comparado las concentraciones de $\mathrm{AMH}$ en mujeres fumadoras y no fumadoras han arrojado resultados contradictorios, los estudios como el actual que han comparado estas concentraciones ajustando por edad han consistentemente encontrado que la declinación de $\mathrm{AMH}$ con la edad se acelera entre las fumadoras ${ }^{16,17}$.

Hasta donde conocemos, este es el primer estudio realizado en mujeres chilenas respecto de la asociación tabaco -concentraciones de AMH. En suma, nuestros hallazgos señalan que el hábito 
de fumar cigarrillos se asocia a concentraciones disminuidas de AMH y esta asociación es compartida por la variable edad. Si embargo la ausencia de una relación dosis respuesta hace difícil una interpretación certera de estos datos. Se necesitan estudios que aclaren los mecanismos que subyacen la asociación descrita.

\section{Referencias}

1. Midgette AS, Baron JA. Cigarette smoking and the risk of natural menopause. Epidemiology 1990; 1 (6): 474-80.

2. Adena MA, Gallagher HG. Cigarette smoking and the age at menopause. Ann Hum Biol 1982; 9 (2): 121-130.

3. Cooper GS, Baird DD, Hulka BS, Weinberg CR, Savitz DA, Hughes CL. Follicle-stimulating hormone concentrations in relation to active and passive smoking. Obstet Gynecol1995; 85 (3): 407-11.

4. Seifer DB, Maclaughlin DT. Müllerian inhibiting substance is an ovarian growth factor of emerging clinical significance. Fertil Steril 2007; 88 (3): 539-46.

5. Jayaprakasan K, Campbell B, Hopkisson J, Johnson I, Raine-Fenning N. A prospective, comparative analysis of anti-müllerian hormone, inhibin-B, and threedimensional ultrasound determinants of ovarian reserve in the prediction of poor response to controlled ovarian stimulation. Fertil Steril2010; 93 (3): 855-64.

6. Kevenaar ME, Meerasahib MF, Kramer P. Serum antimüllerian hormone levels reflect the size of the primordial follicle pool in mice. Endocrinology 2006; 147 (7): 322834.

7. Hehenkamp WJ, Looman CW, Themmen AP, De Jong FH, Te Velde ER, Broekmans FJ. Anti-müllerian hormone levels in the spontaneous menstrual cycle do not show substantial fluctuation. J Clin Endocrinol Metab 2006; 91 (10): 4057-63.

8. Tsepelidis S, Devreker F, Demeestere I, Flahaut A, Gervy C, Englert Y. Stable serum levels of anti-müllerian hormone during the menstrual cycle: a prospective study in normo-ovulatory women. Hum Reprod 2007; 22 (7):
1837-40.

9. Fuentes A, Muñoz A, Barnhart K, Argüello B, Díaz M, Pommer R. Recent cigarette smoking and assisted reproductive technologies outcome. Fertil Steril 2010; 93 (1): 89-95.

10. Fuentes A, Muñoz A, Pommer R, Argüello B, Galleguillos A, Torres A, et al. Decreased anti-Müllerian hormone concentration in follicular fluid of female smokers undergoing artificial reproductive techniques. Chemosphere 2012; 88 (4): 403-6.

11. La Marca A, Giulini S, Tirelli A, Bertucci E, Marsella T, Xella $S$, et al. Anti-mullerian hormone measurement on any day of the menstrual cycle strongly predicts ovarian response in assisted reproductive technology. Hum Reprod 2007; 22 (3): 766-71.

12. Freour T, Masson D, Dessolle L, Allaoua D, Dejoie T, Mirallie S, et al. Ovarian reserve and in vitro fertilization cycles outcome according to women smoking status and stimulation régimen. Arch Gynecol Obstet 2012; 285 (4): 1177-82.

13. La Marca A, Sighinolfi G, Radi D, Argento C, Baraldi E, Carducci A, et al. Anti-Müllerian hormone (AMH) as a predictive marker in assisted reproductive technology (ART). Human Reproduction Update 2010; 16 (2): 113 30.

14. Plante B, Cooper G, Baird D, Steiner A.The impact of smoking on antimüllerian hormone levels in women aged 38 to 50 years. Menopause 2010; 17 (3): 571-6.

15. Freour T, Masson D, Mirallie S, Jean M, Baoh K, Dejoie $\mathrm{T}$, et al. Active smoking compromises IVF outcome and affects ovarian reserve. Reprod Biomed Online 2008; 16 (1): 96-102.

16. Sowers MR, McConnell D, Yosef M, Jannausch ML, Harlow SD, Randolph JF Jr. Relating smoking, obesity, insulin resistance, and ovarian biomarker changes to the final menstrual period.Ann N Y Acad Sci 2010; 1204 (1): 95-103.

17. Seifer DB, Golub ET, Lambert-Messerlian G, Benning L, Anastos K, Watts DH, et al. Variations in serum Müllerian inhibiting substance between white, black, and Hispanic women. Fertil Steril 2009; 92 (5): 1674-8. 